\title{
Gluon perturbative QCD fragmentation function considering the effects of heavy quarkonium mass
}

\section{S. Mohammad Moosavi Nejad*}

Faculty of Physics, Yazd University, P.O. Box 89195-741, Yazd, IRAN

School of Particles and Accelerators, Institute for Research in Fundamental Sciences (IPM),

P.O. Box 19395-5531, Tehran, Iran

E-mail: mmoosavi@yazd.ac.ir

Fragmentation is the dominant production mechanism for heavy-quark-antiquark bound states with large transverse momentum. We analytically calculate the initial scale fragmentation functions (FFs) for a gluon to split into $S$-wave heavy quarkonium state using the perturbative QCD. Our analytical expression of FF depends on the transverse momentum $k_{T}$ of the gluon, and contains most of the kinematical and dynamical properties of the process. The analyses of this paper differ in that we present an analytical form of the transverse momentum dependent FF, using a different approach (Suzuki's model) in comparison with the numerical results presented in other references where the Braaten's model have been used. These $k_{T}$ dependent FFs are necessary to calculate the differential cross sections $d \sigma / d k_{T}$, for which there are experimental data. We also incorporate, for the first time, hadron mass effects in our calculations to improve the FF at the small $z$ regions, where $z$ is the fragmentation parameter. These effects modify the relations between partonic and hadronic variables and reduce the available phase space and are responsible for the low-z threshold.

The European Physical Society Conference on High Energy Physics

22-29 July 2015

Vienna, Austria

${ }^{*}$ Speaker. 


\section{Introduction}

Among all experiments, the production of heavy hadrons have always been interesting. They can be studied to deepen our understanding of strong interaction dynamics. At sufficiently large transverse momentum the production of heavy quarkonia occurs via fragmentation. Fragmentation refers to the process of a parton which carries large transverse momentum so that subsequently decays to form a jet containing the expected hadron and other partons [1]. The quantity which describes this process is the fragmentation function (FF). In order to properly estimate the production rate of a specific quarkonium it is important to obtain the corresponding FF.

Generally, the scattering cross section of the process $A+B \rightarrow H\left(k_{T}\right)+$ Jet, can be expressed as

$$
d \sigma=\sum_{a, b, c} \int_{0}^{1} d x_{a} \int_{0}^{1} d x_{b} \int_{0}^{1} d z f_{a / A}\left(x_{a}, Q\right) f_{b / B}\left(x_{b}, Q\right) d \hat{\sigma}(a+b \rightarrow c+J e t) D_{c}^{H}(z, Q),
$$

where $a$ and $b$ are incident partons in the colliding hadrons, $f_{a / A}$ and $f_{b / B}$ are the parton distribution functions at the scale $Q^{2}$ and $D_{c}^{H}(z, Q)$ is the FF with respect to the scale $Q^{2}$ which can be obtained by evolving from the initial FF $D_{c}^{H}\left(z, Q_{0}\right)$ using the Altarelli-Parisi equations [2].

The particular importance of FFs is for model-independent predictions of the cross sections and decay rates at the Large Hadron Collider (LHC) in which hadrons are detected in the outgoing productions as the colorless bound states.

In [3, 4], we have calculated an exact analytical expression of the initial scale FFs for a heavy quark and gluon to split into S-wave heavy mesons to leading order in the QCD coupling constant, by applying a theoretical approach based on the Suzuki's model [5]. In this elaborate model, Suzuki calculates the heavy FFs considering the convenient Feynman diagrams and the wave function of heavy meson, so the analytical expression of FFs depends on the transverse momentum $k_{T}$ of the initial parton which appears as a phenomenological parameter. The transverse momentum dependent FFs show up explicitly in several semi-inclusive cross sections, especially in azimuthal asymmetries. In the calculation of QCD corrections for these cross sections, the inclusion of transverse momentum dependent FFs is necessary. This is our main motivation to study the $k_{T}$ dependent FFs using the Suzuki's model.

Heavy quarkonia are the simplest particles when the strong interactions are concerned, so that the perturbative QCD approximations to their FFs are well-defined in the nonrelativistic QCD factorization framework [6,7]. In high energy processes, the large contribution of quarkonium production results from gluon fragmentation [8]. This is confirmed by the comparison between the theoretical predictions and the experimental measurements of the heavy quarkonium cross sections. In the present work, we review our previous work [4] and present an analytical form of the transverse momentum dependent FFs for a gluon to fragment into the heavy quarkonium at the leading order of $\alpha_{s}$. Furthermore we, for the first time, impose the effect of finite meson mass on the perturbative QCD FF. We show that how this effect modifies the relations between hadronic and partonic variables and reduces the available phase space.

\section{Theoretical determination of FFs}

In our previous work [4], we focused on the fragmentation of a gluon into the S-wave spinsinglet and spin-triplet heavy quarkonia considering the processes: $g \rightarrow \eta_{c}(=c \bar{c})+g$ and $g \rightarrow$ 
$J / \psi(=c \bar{c})+g g$, and derived an exact analytical form of $D_{g}^{H_{c}}\left(z, \mu_{0}\right)$ using the Suzuki's approach which embeds most of the kinematical and dynamical properties of the process. Here, $z$ refers to the scaled energy variable and $\mu_{0}$ stands for the initial scale of energy. In our calculation, we adopted the infinite momentum frame where the fragmentation parameter in the usual light-cone form $z=\left(E^{H}+k_{L}^{H}\right) /\left(E^{g}+k_{L}^{g}\right)$ is reduced to a more popular form $z=E^{H} / E^{g}$. According to the Lepage-Brodsky's approach [9], we also neglected the relative motion of $Q$ and $\bar{Q}$ therefore we assume, for simplicity, that $Q$ and $\bar{Q}$ are emitted collinearly with each other and they move along the $Z$-axes. Indeed, the Fermi motion of the constituent quarks in the bound state is neglected. The start point was with the definition of FF introduced in [5], as

$$
D_{g \rightarrow H_{c}}(z, \mu)=\int d^{3} \vec{p} d^{3} \vec{k} d^{3} \vec{k}^{\prime}\left|T_{M}\right|^{2} \delta^{3}\left(\overrightarrow{k^{\prime}}+\vec{p}+\vec{k}-\vec{p}^{\prime}\right),
$$

where $\overline{\left|T_{M}\right|^{2}}$ is the average probability amplitude squared of the process and $\vec{p}^{\prime}, \overrightarrow{k^{\prime}}, \vec{p}$ and $\vec{k}$ refer to the momenta of initial and final partons. Also, the probability amplitude $\left(T_{M}\right)$ to split a parton into the meson is expressed as the convolution of the hard scattering amplitude $T_{H}$ which is, in essence, the partonic cross section to produce a heavy quark-antiquark $(Q \bar{Q})$ pair with certain quantum numbers, and the process-independent distribution amplitude $\Phi_{M}$, i.e.

$$
T_{M}=\int\left[d x_{i}\right] T_{H}\left(x_{i}, Q^{2}\right) \Phi_{M}\left(x_{i}, Q^{2}\right),
$$

where $\left[d x_{i}\right]=d x_{1} d x_{2} \delta\left(1-x_{1}-x_{2}\right)$. The short-distance coefficient $T_{H}$ can be calculated as the perturbation series in the strong coupling constant $\alpha_{s}$. The long-distance distribution amplitude $\Phi_{M}$ is the probability amplitude for a $Q \bar{Q}$ pair to evolve into a particular heavy quarkonium state and is related to the mesonic wave function $\Psi_{M}$. By working in the infinite-momentum frame, a simple nonrelativistic wave function is a delta function. The delta function form is convenient for our assumption where we ignore the relative motion of quark and antiquark and thus the constituent quarks are emitted collinearly with each other and they have no transverse momentum. Considering the convenient Feynman diagrams and the Feynman rules, the transition amplitude is calculated and, in conclusion, we obtain the fragmentation function as

$$
D_{g \rightarrow H_{c}}\left(z, \mu_{0}\right)=\frac{N z}{F\left(z,\left\langle k_{T}^{2}\right\rangle\right)}\left\{\left[\frac{z^{2} k_{T}^{2}+M^{2}(1-z)^{2}}{M z(1-z)}-3 m_{c}\right]^{2}-21 m_{c}^{2}\right\}
$$

where,

$$
F\left(z,\left\langle k_{T}^{2}\right\rangle\right)=\left[2 \frac{m_{c}}{M}+\frac{k_{T}^{2}}{M^{2}} \frac{z}{1-z}+\frac{1}{z}-1\right]^{2}\left[\frac{k_{T}^{2}}{M^{2}} \frac{z}{1-z}+\frac{1}{z}-1\right]^{2},
$$

and $N$ is proportional to $\left(\pi C_{F} \alpha_{s} f_{M}\right)^{2}$ but it is related to the normalization condition [10]. Here, for simplicity, we replaced the transverse momentum integration by its average value $\left\langle k_{T}^{2}\right\rangle$, which is a free parameter and can be specified experimentally.

In general, the fragmentation function $D_{g \rightarrow H_{c}}$ depends on both the fragmentation parameter $z$ and the factorization scale $\mu$. The function (2.3) should be regarded as a model for the gluon FF at the scale $\mu_{0}$ of order $2 m_{c}$. For values of $\mu$ much larger than $\mu_{0}$, the obtained FF should be evolved from the scale $\mu_{0}=2 m_{c}$ to the desired scale $\mu$ using the DGLAP equation [2]. In Fig. 1, the FF 
of $g \rightarrow H_{c}$ at the starting scale $\mu_{0}=3 \mathrm{GeV}$ is shown. The behavior of $D_{g}^{H_{c}}$ is shown for different values of the transverse momentum of the gluon.

As a comparison, our result is in good consistency with the FF of Braaten's approach presented in Fig. 3 of Ref. [11] when the non-covariant definition of fragmentation parameter is applied, i.e. $z=\sqrt{M_{H}^{2}+\left(k_{L}^{H}\right)^{2}} / E^{g}=E^{H} / E^{g}$, as we used.

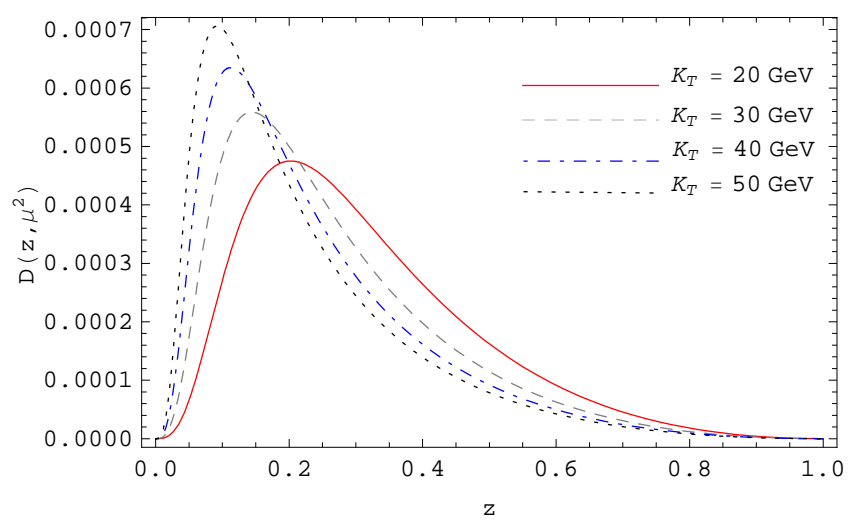

Figure 1: $g \rightarrow H_{c}$ FF as a function of $z$ and $k_{T}$ at the scale $\mu=2 m_{c}$.

\section{Gluon FFs considering mass effects}

In this section, we show how to incorporate the effects of the quarkonium mass $M$ into the relevant kinematic variables of $g \rightarrow H_{c}$ FFs, a topic with a very little attention paid to in the literature. The FF depends on the fragmentation parameter $z$ while there is some freedom in defining this parameter when the hadron mass is considered. In Ref. [11], using the covariant and non-covariant definitions of the parameter $z$ authors analyzed the uncertainties induced by different definitions of the momentum fraction $z$ in the application of gluon to heavy quarkonium FF. Here, we review the different definitions of the fragmentation parameter defined in the various Lorentz boost invariant forms, i.e.
a) $z=\frac{E^{H}+k_{L}^{H}}{E^{g}+k_{L}^{g}}$
b) $z=\frac{k^{g} \cdot k^{H}}{\left(k^{g}\right)^{2}}$
c) $z=\frac{\sqrt{M_{H}^{2}+\left(k_{L}^{H}\right)^{2}}}{E^{g}}$,
d) $z=\frac{\sqrt{M_{H}^{2}+\left(k_{L}^{H}\right)^{2}}+k_{L}^{H}}{E^{g}+k_{L}^{g}}$,
e) $z=\frac{k_{L}^{H}}{k_{L}^{g}}$

In the above equations we take the $\mathrm{Z}$-axis along the momentum of outgoing meson and $E^{g}, k_{L}^{g}, E^{H}$ and $k_{L}^{H}$ are the energies and the longitudinal components (Z-components) of the four-momenta of the fragmenting gluon and the produced heavy quarkonium $H$, respectively. The first definition (3.1a) is the usual light-cone form and this definition along with the second definition are hard to be employed in the application of the gluon FFs, because they involve the longitudinal momentum of the resulting heavy quarkonium. Instead, usually the non-covariant definitions (Eqs. (3.1c)-(3.1e)) are used approximately, which are convenient for the finite momentum frame. In Ref. [11], authors 
have showed that the FFs have strong dependence on the gluon momentum $\vec{k}$, and when $|\vec{k}| \rightarrow \infty$ these FFs approach to the FF in the light-cone definition (3.1a) and large uncertainties remain while the non-covariant definitions of $z$ are employed.

In our previous calculation we applied a custom definition of the fragmentation parameter as $z=E^{H} / E^{g}$, a special choice which is convenient for the case of massless parton and hadron which takes the values of $0 \leq z \leq 1$. To incorporate the effects of the hadron mass into the fragmentation function a suitable choice of scaling variable is the usual light-cone form (3.1a). This form of the fragmentation parameter is invariant with respect to boosts along the three-axis. This axis was considered as the flight direction of outgoing meson.

Considering the light-cone form of the fragmentation parameter (3.1a) and Fig. 4 of Ref. [4], the scaling variable reads

$$
\eta=\frac{E^{H}+k_{L}^{H}}{E^{g}+k_{L}^{g}}=\frac{\bar{P}_{0}+\bar{P}_{L}}{p_{0}+p_{L}}
$$

Considering the infinite momentum frame where $p_{0} \approx p_{L}$ and the definition of the fragmentation parameter in the absence of meson mass $\left(z=E^{H} / E^{g}\right)$, one has

$$
\eta=\frac{z P_{0}+\sqrt{z^{2} P_{0}^{2}-M^{2}}}{2 p_{0}}
$$

where $M$ stands for the meson mass. At the fragmentation process with the sufficiently large transverse momentum, one can write $m_{g}^{2} \approx p_{0}{ }^{2}-k_{T}^{2}$. Since the gluon is considered as an on-shell particle then $p_{0}=k_{T}$.

Now, to obtain the FF in the presence of quarkonium mass we start with the initial definition of fragmentation [10], i.e. $D_{Q \rightarrow H}(z)=1 / \sigma \cdot d \sigma(z) / d z$. As a generalization of the massless quarkonium case, we assume that the cross section which we have been computing is $d \sigma(\eta) / d \eta$ which is related to the measured observable $d \sigma(z) / d z$ as $d \sigma(z) / d z=d \sigma(\eta) / d \eta \times d \eta / d z$ where one has $d \eta / d z=\left(1-M^{2} /\left(2 \eta k_{T}\right)^{2}\right)^{-1}$. Our new scaling variable is related to the old one $z$ as

$$
\eta=\frac{z}{2}\left\{1+\sqrt{1-\left(\frac{M}{z k_{T}}\right)^{2}}\right\} .
$$

Now the observable quantity $D_{g \rightarrow H}(z)$ is expressed as

$$
D_{g \rightarrow H}(z, \mu)=\frac{1}{1-\frac{M^{2}}{4 \eta^{2} k_{T}^{2}}} D_{g \rightarrow H}(\eta(z), \mu) .
$$

Now, $D_{g \rightarrow H}(\eta, \mu)$ is the FF obtained in (2.3) by substituting $z \rightarrow \eta$ and the kinematically allowed $z$ ranges are $M / k_{T}<z \leq 1$. In Fig. 2 the behavior of $g \rightarrow H_{c}$ FF is shown for the massless and massive meson taking $m_{Q}=m_{c}=1.5 \mathrm{GeV}$ and $M=m_{J / \psi}=3096.916 \pm 0.011 \mathrm{MeV}$ [12]. As is seen the effect of quarkonium mass increases the size of FF at low values of $z$ and the peak position is shifted towards higher values of $z$ and it also creates a threshold at $z=0.155$.

\section{Conclusion}

The dominant production mechanism for heavy quarkonium at the high transverse momentum is fragmentation. In this work, we reviewed our previous work [4] and gave out an analytical form 


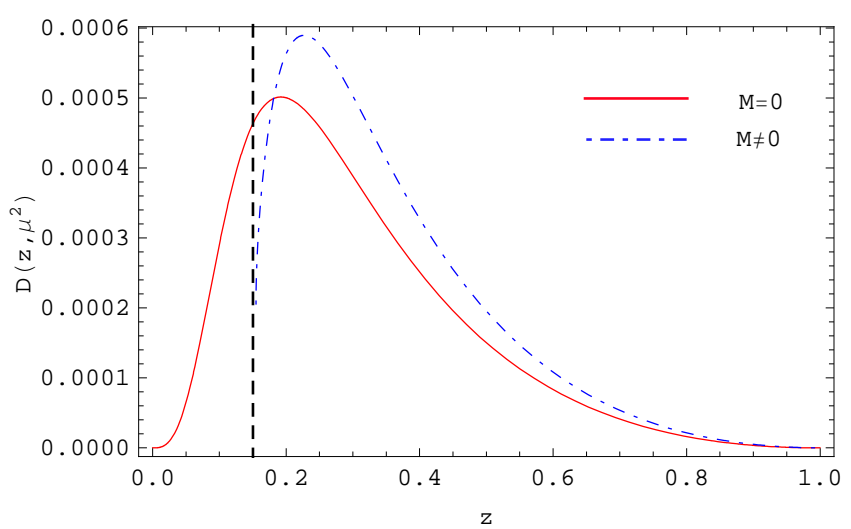

Figure 2: $g \rightarrow H_{c}$ FF at $\mu_{0}=2 m_{c}$ as a function of $z$ when $M=0$ (solid line) and $M \neq 0$ (dot-dashed line) taking $k_{T}=20 \mathrm{GeV}$. Threshold at $z=0.155$ is also shown.

for the initial FF of gluon to split into S-wave quarkonium states to leading order in $\alpha_{s}$, using the nonrelativistic QCD factorization framework. Our result depends on the transverse momentum of the gluon and is in good agreement with the result presented in Ref. [11] when one use the normal definition of the fragmentation parameter, i.e. $z=E^{H} / E^{g}$. We also studied, for the first time, heavy quarkonium mass corrections on the perturbative QCD FFs and their theoretical uncertainty due to the freedom in the choice of the fragmentation parameter. As it is seen these corrections are responsible for the low-z threshold.

Finally, although the results presented in this work are schematically for charmonium, but they can be directly applied to the S-wave bottomonium sate $\Upsilon$ except that $m_{c}$ is replaced by $m_{b}$ and the decay constant $f_{M}$ is the appropriate constant for the bottomonium mesons.

\section{References}

[1] E. Braaten and T. C. Yuan, Phys. Rev. Lett. 71 (1993) 1673.

[2] G. Altarelli and G. Parisi, Nucl. Phys. B126, 298 (1977).

[3] S. M. M. Nejad and A. Armat, Eur. Phys. J. Plus 128 (2013) 121.

[4] S. M. Moosavi Nejad, Eur. Phys. J. Plus 130 (2015) 7, 136.

[5] M. Suzuki, Phys. Lett. B 71 (1977) 139.

[6] G. T. Bodwin, E. Braaten and G. P. Lepage, Phys. Rev. D 51 (1995) 1125.

[7] W. E. Caswell and G. P. Lepage, Phys. Lett. B 167 (1986) 437.

[8] D. P. Roy and K. Sridhar, Phys. Lett. B 339 (1994) 141.

[9] G. P. Lepage and S. J. Brodsky, Phys. Rev. D 22 (1980) 2157.

[10] M. Suzuki, Phys. Rev. D 33 (1986) 676.

[11] W. Qi, C. F. Qiao and J. X. Wang, Phys. Rev. D 75 (2007) 074012.

[12] K. Nakamura et al. (Particle Data Group), J. Phys. G 37, 075021 (2010). 